\title{
WSN-Based Height Estimation of Moving Object in Surveillance Systems
}

\author{
Jaeseok Shim ${ }^{1}$ and Yujin Lim ${ }^{2}$ \\ ${ }^{1}$ Department of Computer Science, University of Suwon, San 2-2, Wau-ri, Bongdam-eup, Hwaseong, \\ Gyeonggi-do 445-743, Republic of Korea \\ ${ }^{2}$ Department of Information Technology Engineering, Sookmyung Women's University, Cheongpa-ro 47-gil 100, \\ Yongsan-gu, Seoul 04310, Republic of Korea
}

Correspondence should be addressed to Yujin Lim; yujin91@sookmyung.ac.kr

Received 17 August 2016; Accepted 22 September 2016

Academic Editor: Hyun-Ho Choi

Copyright (C) 2016 J. Shim and Y. Lim. This is an open access article distributed under the Creative Commons Attribution License, which permits unrestricted use, distribution, and reproduction in any medium, provided the original work is properly cited.

In the WSN- (wireless sensor network-) based surveillance system to detect undesired intrusion, all detected objects are not intruders. In order to reduce false alarms, human detection mechanism needs to determine if the detected object is a human. For human detection, physical characteristics of human are usually used. In this paper, we use the physical height to differentiate an intruder from detected objects. Using the measured information from sensors, we estimate the height of the detected object. Based on the height, if the detected object is decided as an intruder, an alarm is given to a control center. The experimental results indicate that our mechanism correctly and fast estimates the height of the object without complex computation.

\section{Introduction}

Conventional surveillance systems address a detection problem of an abnormal event in monitored space by deploying video systems. However, video systems are expensive in terms of hardware, storage, and communications. In particular, collecting multiple video streams imposes high demands on storage, online monitoring, and video analysis. The WSN (wireless sensor network) technology provides means to develop such a system with low costs [1]. The WSN can run standalone or it can be used to trigger external surveillance system like a video system. For example, in a hybrid system, when WSN detects and notifies an abnormal event, WSN triggers a video surveillance system. Then, camera is activated and zooms into the monitored space. The hybrid system optimizes accuracy, while keeping costs low. WSN is ideal for monitoring application because it is a fully automated system which does not require any human intervention and easily connected to external system so it can notify the user of the undesired situation.

There are a lot of monitoring applications based on WSN technology and they are interested in monitoring specific events or objects [2-5]. Not only do they need to figure out occurrence or existence of events or objects, but also they need to find out where the events have occurred or the objects are present. Examples of that kind of applications include fire monitoring systems, surveillance systems, livestock monitoring, and protection systems. The target application aimed by this paper is surveillance systems for home, office, or factory. In surveillance systems, it is very important to detect undesired intrusion while nobody is present in office, home, or factory. Typically, WSN-based surveillance systems start with motion detecting. Once any motion in the monitored space is detected, because all moving objects are not intruders, human detection mechanism needs to determine if the detected object is a human in order to reduce false alarms to video system. For human detection, physical characteristics of human are usually used. Height, speed, and body heat are one of these physical characteristics. When the height of a detected object is within a reasonable range for normal adults, for example, between $150 \mathrm{~cm}$ and $200 \mathrm{~cm}$, we can consider the object as a human. In the same manner, we can check whether the mobility speed or the body heat of the object is within a reasonable range. In this paper, we use the physical height to differentiate an intruder from detected objects $[6,7]$. Using the ranging information from sensors, we present a 
height estimation mechanism for the detected object [8]. In order to reduce the estimation error of the height of the detected object, we extend the height estimation mechanism with a compensation algorithm using interpolation method. If the finally estimated height is in the height range for normal adults, it is determined that the object is a human and an alarm is given to activate video surveillance system. Our mechanism makes WSN-based surveillance system selfconfiguring because it can configure a range for normal adults in a dynamic way.

The rest of the paper is organized as follows. In Section 2, we introduce the related studies for $3 \mathrm{D}$ localization. In Section 3, we propose a height estimation mechanism with an error compensation algorithm for human detection. Then, we show the performance of the proposed mechanism in Section 4. Finally, the conclusion remarks are given in Section 5.

\section{Related Work}

In a localization system, most algorithms work on 2D (2dimensional) plane, that is, $x$ and $y$ plane. In the 2D localization algorithms, the process of estimation is less complex and faster than the process of 3D (3-dimensional) localization algorithms [9]. The 2D localization algorithms provide good accuracy on flat terrains but they are difficult to estimate on rough terrains. Thus, mapping these positions estimated by the $2 \mathrm{D}$ algorithms to the real world can cause errors. By using $3 \mathrm{D}$ with one extra plane, that is, $z$ plane, $3 \mathrm{D}$ localization algorithms provide more accurate results on rough terrains. In $2 \mathrm{D}$ space, at least three anchor nodes determine a coordinate system. In 3D space, four anchor nodes at least are required.

3D DV-Hop algorithm [10] expands the traditional rangefree DV-Hop algorithm into 3D space. In the algorithm, the minimum hop counts between the detected object and the anchor nodes are calculated by using mobile agents. Then, the algorithm calculates the average per-hop distance of the object by using the minimum hop counts and the average perhop distance of the anchor nodes. Based on the calculated distance, the position of the object is estimated. 3D Centroid algorithm [11] expands the traditional Centroid algorithm. All anchor nodes send their position information within their transmission range. An object collects the signals from anchor nodes and it selects randomly four anchor nodes in range to form a series of tetrahedrons. For each tetrahedron, the algorithm calculates the barycenter. Finally, the average coordinate of these barycenters is used as the estimated position of the object.

In 3D-ADAL (3-dimensional azimuthally defined area localization algorithm) [12], a mobile beacon moves in the whole network and broadcasts beacon messages within its transmission range. First, each sensor node estimates its position in the local $x y$-plane. Then, each node focuses on the local $y z$-plane of the mobile beacon and determines its altitude by using the information of tilt in the beacon message. In [13], the loss of wireless signal strength between the object and an anchor node is converted to the distance first, and then the maximum-likelihood estimation method is used to calculate the $3 \mathrm{D}$ coordinate of the object.
In other words, RSSI (received signal strength indication) value between the object and anchor node is converted to the distance. For $3 \mathrm{D}$ positioning, the object needs to communicate with four adjacent anchor nodes at least. When obstacles are present, the attenuation of wireless signal does not meet linear relationship with the distance any longer, so the compensation algorithm like the maximum-likelihood estimation method is used.

In 3D UMP (unitary matrix pencil) [14], it is based on TOA (time-of-arrival) estimation of UWB (ultrawideband) signal using UMP algorithm. The UMP algorithm is proposed to measure the distance between two nodes. The algorithm is extended to the applications of UWB-based WSN to reduce computational load and improve time resolution. The estimation results are used by multilateral localization algorithm for $3 \mathrm{D}$ position computation. In SDI (space distance intersection) [15], each sensor node measures a set of distances with the mobile beacon by using TOA techniques of UWB signal.

In [16], a closed-form solution for 3D localization using AOA (angle of arrival) is presented. It reduces the amount of estimation bias that is caused by the measurement noise and sensor position errors. Besides it achieves asymptotically the CRLB (Cramer-Rao lower bound) performance and maintains a bias level close to the maximum-likelihood estimator. Reference [17] applies the WIV (weighted instrumental variable) technique for AOA positioning. The WIV technique uses an IV matrix to reduce the noise correlation between the regressor and regressand.

The conventional 3D localization techniques require at least four anchor nodes to calculate the position of the detected object. Besides, they have high processing overhead. However, our surveillance system needs the only height information of the detected object to determine if the object is human, instead of the position of the object. Thus, we propose a lightweight height estimation mechanism with low processing overhead and our mechanism requires at least three anchor nodes. Based on the height estimation of the object, we can implement fast and accurate human detection system.

\section{Proposed Mechanism}

In order to estimate the height of a target object, we firstly estimate the distance from a ceiling to the target. Given the distance, the height of the target is calculated by subtracting the distance from the height of the monitored space where the target locates. We assume that sensors are deployed on a ceiling in the monitored space. The sensors measure the distances from themselves to the target. In Figure 1, the base of upside-down triangular pyramid indicates a ceiling of the monitored space. Three sensors mounted on a ceiling are vertices $A, B$, and $C$. The target is located at the apex $\left(P_{x}\right)$ of the pyramid. The distances measured by sensors $A, B$, and $C$ are $d_{x}, e_{x}$, and $f_{x}$. We propose an algorithm to estimate the height of the pyramid, $x$. Given $a, b, c, d_{x}, e_{x}$, and $f_{x}$, our algorithm estimates the height $x$.

The base area is the area of the triangular base in the pyramid. Using Heron's formula, we calculate the area of a 


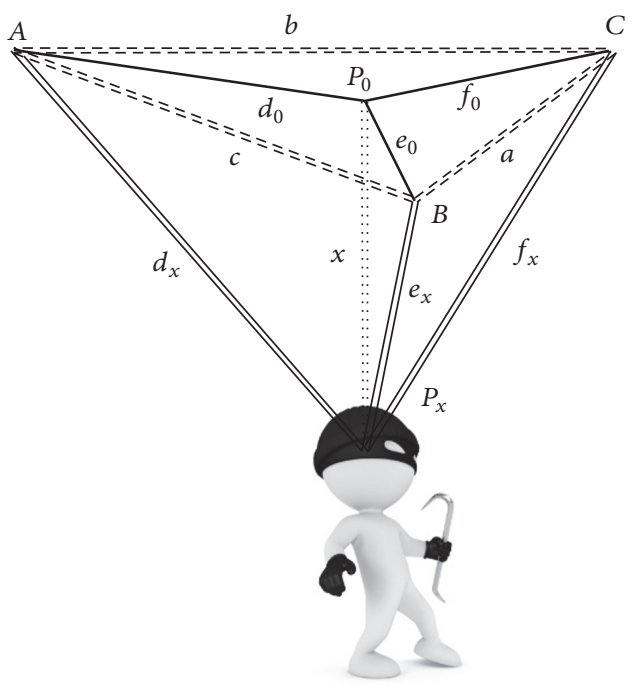

FIGURE 1: Symbols used by our algorithm.

triangle, $S(a b c)$ when the lengths of all three sides, $a, b$, and $c$ are known [18]. The base area whose sides have lengths $a, b$, and $c$ is calculated using Heron's formula, as below.

$$
\begin{aligned}
& S(a b c) \\
& \quad=(a+b+c)(-a+b+c)(a-b+c)(a+b-c) .
\end{aligned}
$$

In other words, the base area with three vertices $A, B$, and $C$ is

$$
\begin{aligned}
\text { Afrea }_{\text {Base }} & =\sqrt{S(a b c)} \\
& =\sqrt{S\left(a e_{0} f_{0}\right)}+\sqrt{S\left(b f_{0} d_{0}\right)}+\sqrt{S\left(c d_{0} e_{0}\right)} .
\end{aligned}
$$

The lateral area is the sum of areas of the side faces, that is, $\triangle B C P_{x}, \triangle C A P_{x}$, and $\triangle A B P_{x}$, in the pyramid. The lateral area is

$$
\text { 4Area } \text { Lateral }=\sqrt{S\left(a e_{x} f_{x}\right)}+\sqrt{S\left(b f_{x} d_{x}\right)}+\sqrt{S\left(c d_{x} e_{x}\right)} .
$$

The height of the pyramid $x$ meets the base at a point, called $P_{0}$ (we can find $P_{0}$ by using triangulation). Using $d_{0}, e_{0}$, and $f_{0}$ in $d_{x}^{2}=d_{0}^{2}+x^{2}, e_{x}^{2}=e_{0}^{2}+x^{2}$, and $f_{x}^{2}=f_{0}^{2}+x^{2},(3)$ is rewritten as follows:

$$
\begin{aligned}
\text { AArea }_{\text {Lateral }}= & \sqrt{S\left(a e_{0} f_{0}\right)+4 a^{2} x^{2}} \\
& +\sqrt{S\left(b f_{0} d_{0}\right)+4 b^{2} x^{2}} \\
& +\sqrt{S\left(c d_{0} e_{0}\right)+4 c^{2} x^{2}} .
\end{aligned}
$$

By treating $S\left(a e_{0} f_{0}\right), S\left(b f_{0} d_{0}\right)$, and $S\left(c d_{0} e_{0}\right)$ as constants, (4) is rewritten as follows:

$$
\begin{aligned}
& \sqrt{S\left(a e_{0} f_{0}\right)+4 a^{2} x^{2}}+\sqrt{S\left(b f_{0} d_{0}\right)+4 b^{2} x^{2}} \\
& +\sqrt{S\left(c d_{0} e_{0}\right)+4 c^{2} x^{2}}>\sqrt{4 a^{2} x^{2}}+\sqrt{4 b^{2} x^{2}} \\
& +\sqrt{4 c^{2} x^{2}}=2(a+b+c) x .
\end{aligned}
$$

Using $\sqrt{a+b} \leq \sqrt{a}+\sqrt{b}(a, b \geq 0)$, we rewrite (5) as follows:

$$
\begin{aligned}
& \sqrt{S\left(a e_{0} f_{0}\right)+4 a^{2} x^{2}}+\sqrt{S\left(b f_{0} d_{0}\right)+4 b^{2} x^{2}} \\
& +\sqrt{S\left(c d_{0} e_{0}\right)+4 c^{2} x^{2}} \leq \sqrt{S\left(a e_{0} f_{0}\right)}+\sqrt{4 a^{2} x^{2}} \\
& +\sqrt{S\left(b f_{0} d_{0}\right)}+\sqrt{4 b^{2} x^{2}}+\sqrt{S\left(c d_{0} e_{0}\right)}+\sqrt{4 c^{2} x^{2}} \\
& \quad=2(a+b+c) x+\sqrt{S(a b c)} .
\end{aligned}
$$

Thus, the lateral area is

$$
\begin{aligned}
\frac{1}{2}(a+b+c) x & <\text { Area }_{\text {Lateral }} \\
& \leq \frac{1}{2}(a+b+c) x+\frac{1}{4} \sqrt{S(a b c)} .
\end{aligned}
$$

We define the function $f(x)$ as follows:

$$
\begin{aligned}
& f(x)=\frac{1}{4}\left(\sqrt{S\left(a e_{0} f_{0}\right)+4 a^{2} x^{2}}+\sqrt{S\left(b f_{0} d_{0}\right)+4 b^{2} x^{2}}\right. \\
& \left.+\sqrt{S\left(c d_{0} e_{0}\right)+4 c^{2} x^{2}}\right) .
\end{aligned}
$$

Using (7), we define the function $g(x)$ to estimate the lateral area as follows:

$$
g(x)=\frac{1}{2}(a+b+c) x
$$

We can estimate $x_{\text {est }}$ when $g\left(x_{\text {est }}\right)=f(x)$ as follows:

$$
\begin{aligned}
x_{\text {est }} & =\frac{2 f(x)}{a+b+c} \\
& =\frac{\sqrt{S\left(a e_{x} f_{x}\right)}+\sqrt{S\left(b f_{x} d_{x}\right)}+\sqrt{S\left(c d_{x} e_{x}\right)}}{2(a+b+c)} .
\end{aligned}
$$

The estimation error is the difference between $f(x)$ and $g(x)$. By tracking limit as $x$ to infinity, the estimation error converges to 0 as follows:

$$
\lim _{x \rightarrow \infty}(f(x)-g(x)) \approx 0
$$

But, when $x=0$, the error is maximized

$$
f(0)-g(0)=\frac{1}{4} \sqrt{S(a b c)} .
$$

To reduce the estimation error, we improve (7) using $\sqrt{a+b} \leq \sqrt{a}+\sqrt{b}(a, b \geq 0)$ as follows:

$$
\begin{aligned}
\frac{1}{2}( & a+b+c) x+\frac{1}{4} \sqrt{S(a b c)} \\
= & \frac{1}{4}\left(\sqrt{S(a b c)}+\sqrt{4(a+b+c)^{2} x^{2}}\right) \\
\geq & \frac{1}{4} \sqrt{S(a b c)+4(a+b+c)^{2} x^{2}} .
\end{aligned}
$$




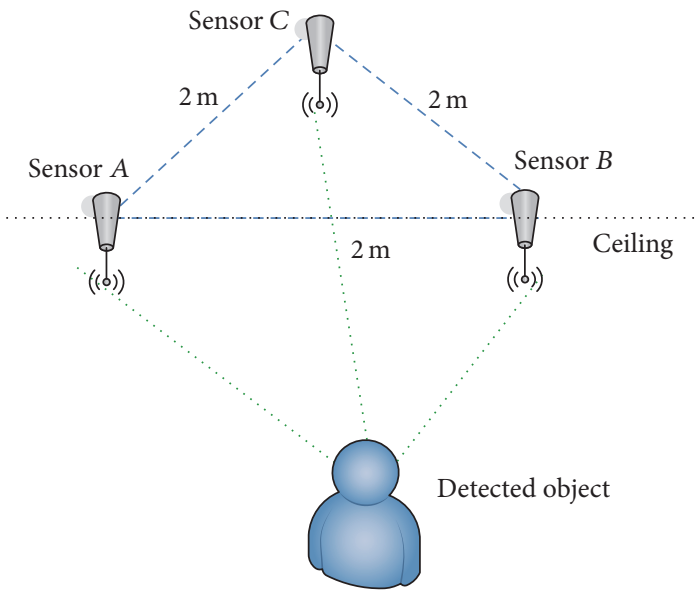

(a) Test environment 1

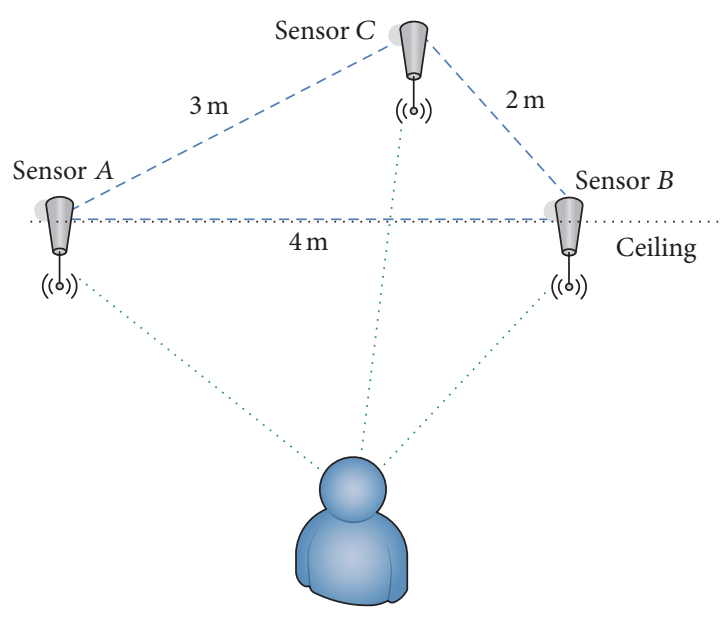

(b) Test environment 2

FIGURE 2: Test environments for evaluating performance.

Using (13), we improve the function $g(x)$ to estimate the lateral area as follows:

$$
h(x)=\frac{1}{4} \sqrt{S(a b c)+4(a+b+c)^{2} x^{2}} .
$$

Using (14), we can estimate $x_{\text {est_new }}$ when $h\left(x_{\text {est_new }}\right)=f(x)$ as follows:

$$
\begin{aligned}
& x_{\text {est_new }}=\frac{\sqrt{16 f(x)^{2}-S(a b c)}}{2(a+b+c)} \\
& =\frac{\sqrt{\left(\sqrt{S\left(a e_{x} f_{x}\right)}+\sqrt{S\left(b f_{x} d_{x}\right)}+\sqrt{S\left(c d_{x} e_{x}\right)}\right)^{2}-S(a b c)}}{2(a+b+c)} .
\end{aligned}
$$

As (7), we can get the following relationship:

$$
\begin{aligned}
\frac{1}{2}(a+b+c) x & <h(x) \\
& \leq \frac{1}{2}(a+b+c) x+\frac{1}{4} \sqrt{S(a b c)}
\end{aligned}
$$

Finally, when $x$ increases on infinity or decreases on 0 , the estimation error converges to 0 as follows:

$$
\begin{array}{r}
\lim _{x \rightarrow \infty}(f(x)-h(x)) \approx 0, \\
f(0)-h(0)=0 .
\end{array}
$$

As a result, given $a, b, c, d_{x}, e_{x}$, and $f_{x}$, we estimate the height of the target by using (15) while minimizing the estimation error.

\section{Performance Analysis}

To evaluate the performance of our mechanism, we implement sensors on the mote modules [19] equipped with DMS (distance measurement sensor) and communications between them are based on IEEE 802.15.4 standard [20]. We deploy three sensors on a ceiling in a laboratory in our college building and we devise two test environments, as shown in Figure 2. Firstly, Figure 2(a) shows the test environment 1. In the environment, we use an equilateral triangle which is a triangle that has equal length $(2 \mathrm{~m})$ on all three sides. Figure 2(b) shows the test environment 2 . We use a triangle with $2 \mathrm{~m} \times 3 \mathrm{~m} \times 4 \mathrm{~m}$. We have two test scenarios for each test environment. In the first scenario, there is no obstacle between sensors and the detected object. In the second scenario, there is an obstacle, such as ceiling fixture, between sensor $A$ and the detected object. In both test environments, we set the distance between the ceiling and a detected object $(x)$ to $0.5 \mathrm{~m}$ or $1 \mathrm{~m}$, respectively. For each experiment, we measure 1000 samples.

Figure 3 shows the estimation accuracy of our mechanism in the test environment 1 . We plot a normal distribution with mean $\mu$ and standard deviation $\sigma$. Each dot indicates $x_{\text {est_new }}$ calculated by using (15) based on each measured sample. Figures 3(a) and 3(b) indicate the estimation accuracy when there is no obstacle between the sensors and the object. In the subfigures, $\pm 2 \sigma$ shows a $95 \%$ confidence interval. When $x=0.5 \mathrm{~m}, 946$ samples are correctly estimated as $x \approx$ $x_{\text {est_new }}$, and the estimation accuracy is $94.6 \%$. When $x=$ $1 \mathrm{~m}$, the accuracy is $95 \%$. Besides, we are $99 \%$ confident that the estimation accuracies are $99.6 \%$ and $99.5 \%$, respectively. Figures 3(c) and 3(d) indicate the estimation accuracy when there is the obstacle. In the subfigures, the effect of an obstacle on the estimation is analyzed. When $x=0.5 \mathrm{~m}$, the estimation accuracy is $91.2 \%$ or $99 \%$ with a $95 \%$ or 99\% level of confidence, respectively. When $x=1 \mathrm{~m}$, the accuracy is $94.6 \%$ or $99.4 \%$. The performance slightly decreases due to the environmental impact such as obstacles. To compensate the impact, more sensors could be deployed or error compensation mechanism could be used.

Figure 4 shows the estimation accuracy of our mechanism in the test environment 2. The figure shows the effect of the deployment of sensors on the estimation. As shown 


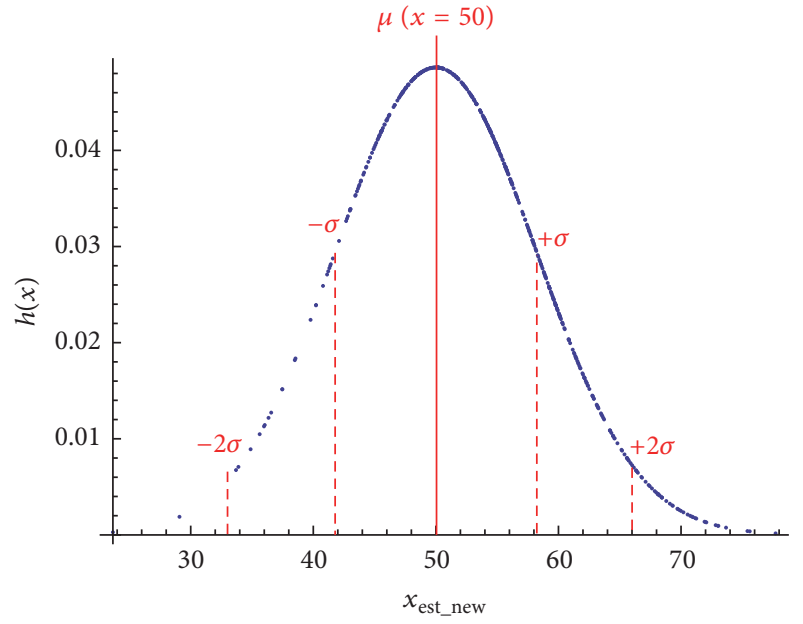

(a) $x=0.5 \mathrm{~m}$, no obstacle

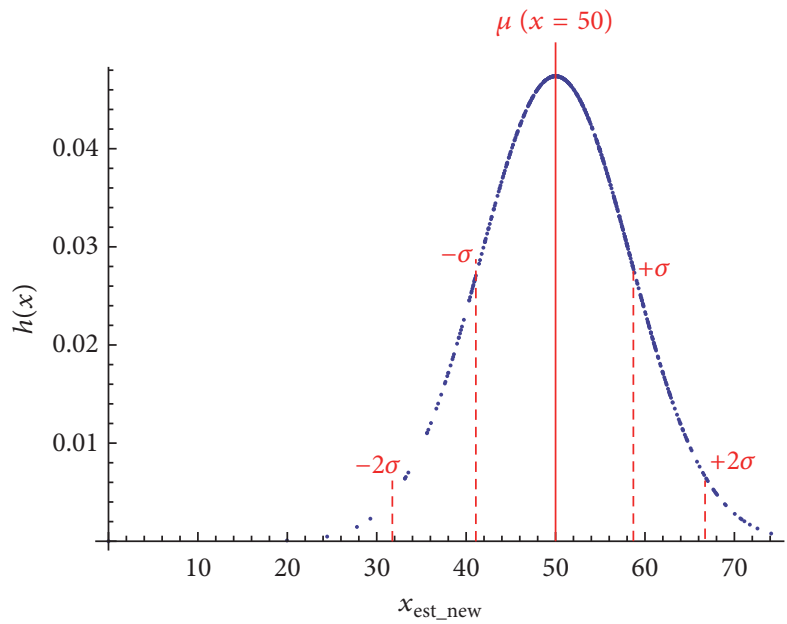

(c) $x=0.5 \mathrm{~m}$, obstacle

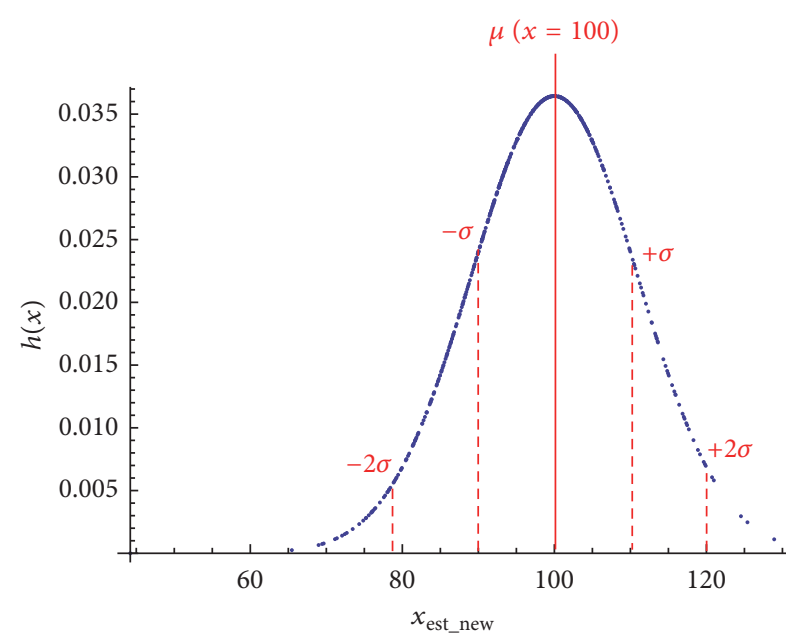

(b) $x=1 \mathrm{~m}$, no obstacle

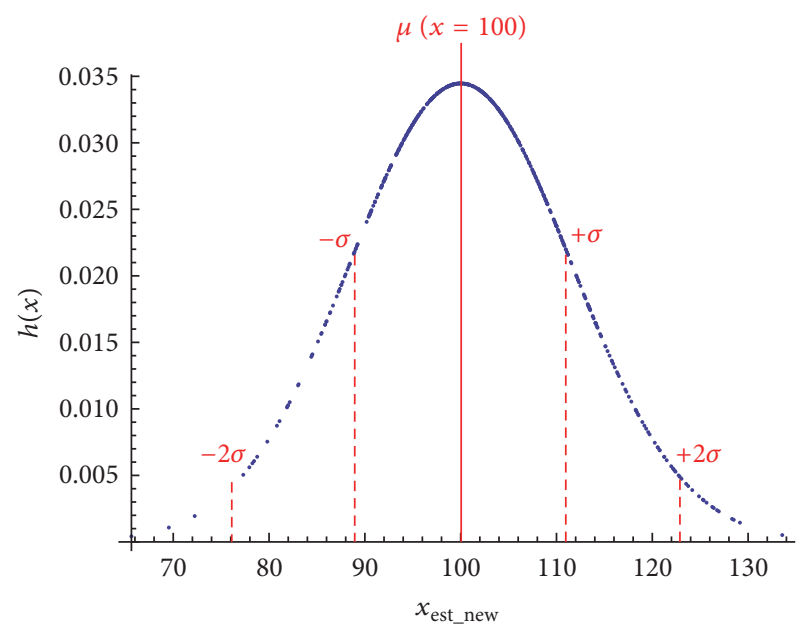

(d) $x=1 \mathrm{~m}$, obstacle

FIGURE 3: Estimation accuracy in test environment 1.

in Figures 4(a) and 4(b), when there is no obstacle between the sensors and the object, the accuracies are $95.6 \%$ and $94.8 \%$ with a $95 \%$ level of confidence. Besides, we are $99 \%$ confident that the accuracies are $98.8 \%$ and $99.2 \%$. When $x=0.5 \mathrm{~m}$ with the obstacle, as shown in Figure 4(c), the estimation accuracy is $93.4 \%$ or $98.8 \%$ with a $95 \%$ or $99 \%$ level of confidence, respectively. When $x=1 \mathrm{~m}$ with the obstacle, as shown in Figure 4(d), the accuracy is $89.6 \%$ or $99.2 \%$.

Figure 5 shows the estimation error of our mechanism in the test environment 2 with the obstacle. In the figure, the effect of the number of sensors on the estimation is analyzed. Because our mechanism operates based on the area of a triangle, we increase the number of sensors from 3 to 5 when $x=1 \mathrm{~m}$. In the figure, estimation error $0 \%$ means that the height of the object is estimated exactly. We measure 100 samples for the figure and the $x$-axis indicates the number of the experimental samples. As shown in the figure, the accuracy is about $99 \%$ when three sensors are deployed. When four or five sensors are deployed, we randomly select three sensors to form a series of triangles. For each triangle, our mechanism estimates the distance. Then, the average distance of the estimated distances is used as the final estimated distance between the ceiling and the object. The estimation accuracies are over $99 \%$ when four or five sensors are deployed. As a result, the performance is more affected by obstacle than the deployment of sensors or the number of sensors. The experimental results indicate that our mechanism correctly and fast estimates the distance from a ceiling to an object without complex computation.

\section{Conclusion}

In the WSN-based surveillance systems, they need to figure out the occurrence or existence of events or objects. To estimate the position of the events or objects, 3D localization techniques have been used. However, the conventional 3D localization techniques require at least four anchor nodes to calculate the position of the detected object. Besides, they cause high processing overhead. However, our surveillance system needs the only height information of the detected 


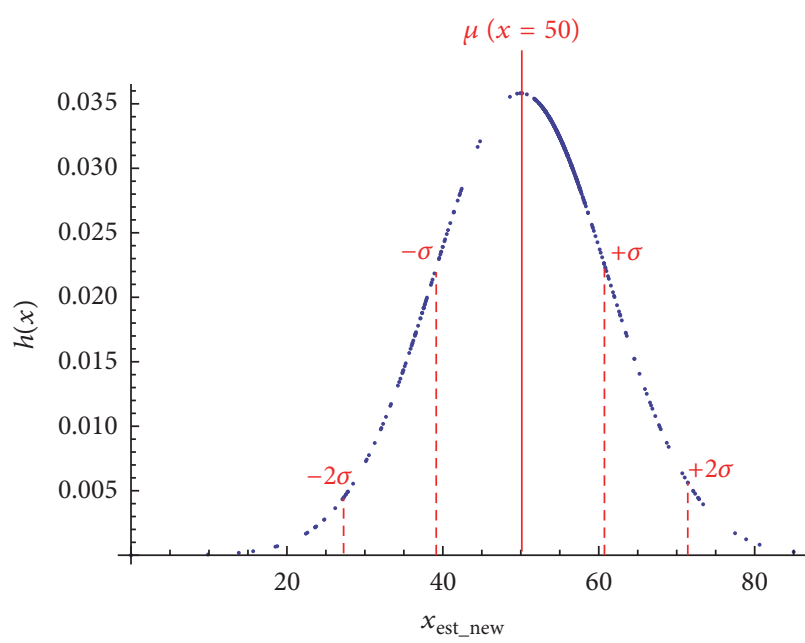

(a) $x=0.5 \mathrm{~m}$, no obstacle

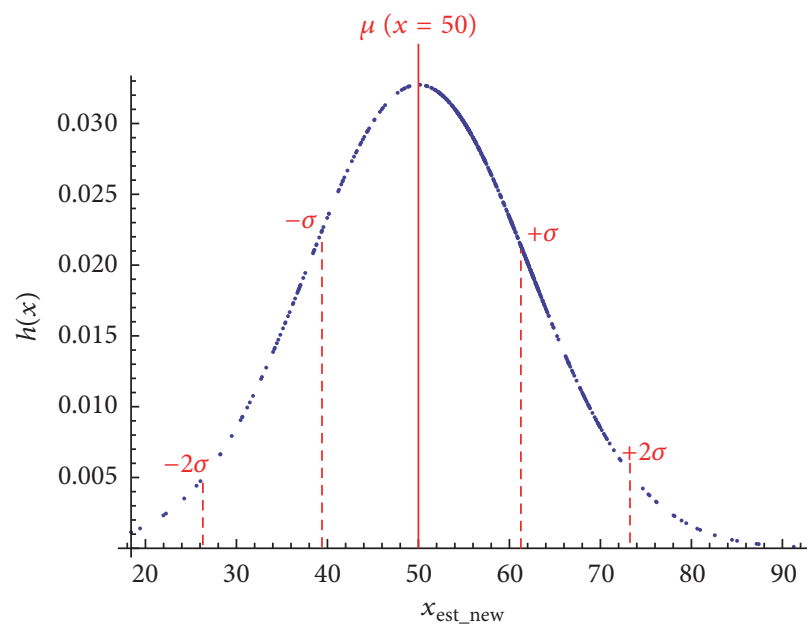

(c) $x=0.5 \mathrm{~m}$, obstacle

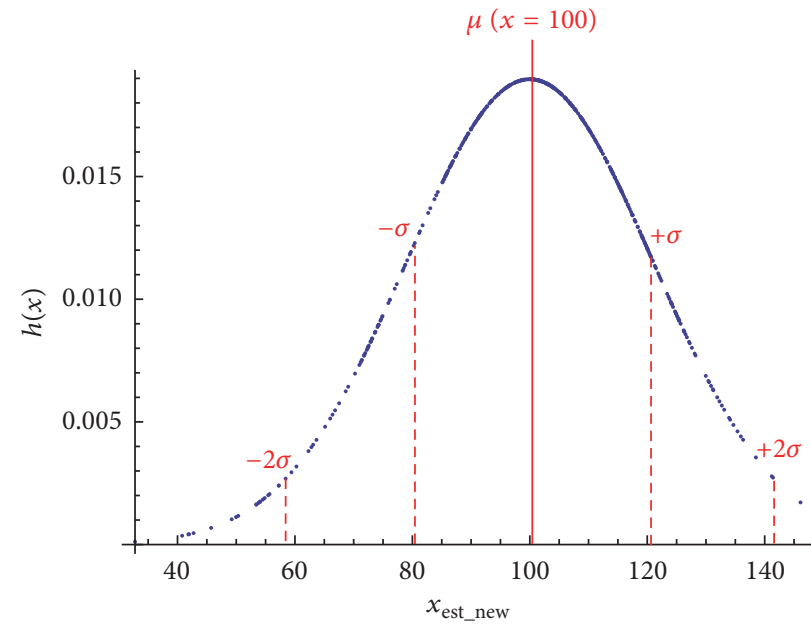

(b) $x=1 \mathrm{~m}$, no obstacle

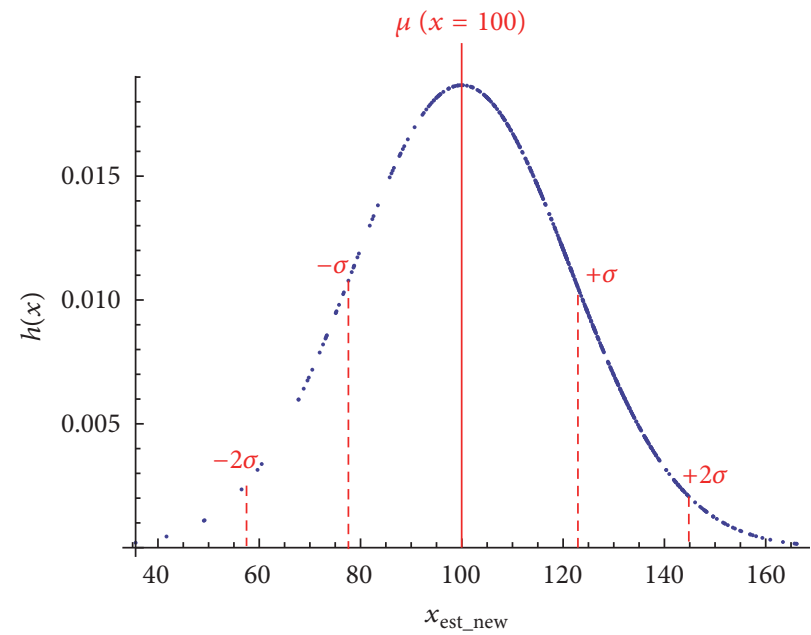

(d) $x=1 \mathrm{~m}$, obstacle

FIGURE 4: Estimation accuracy in test environment 2.

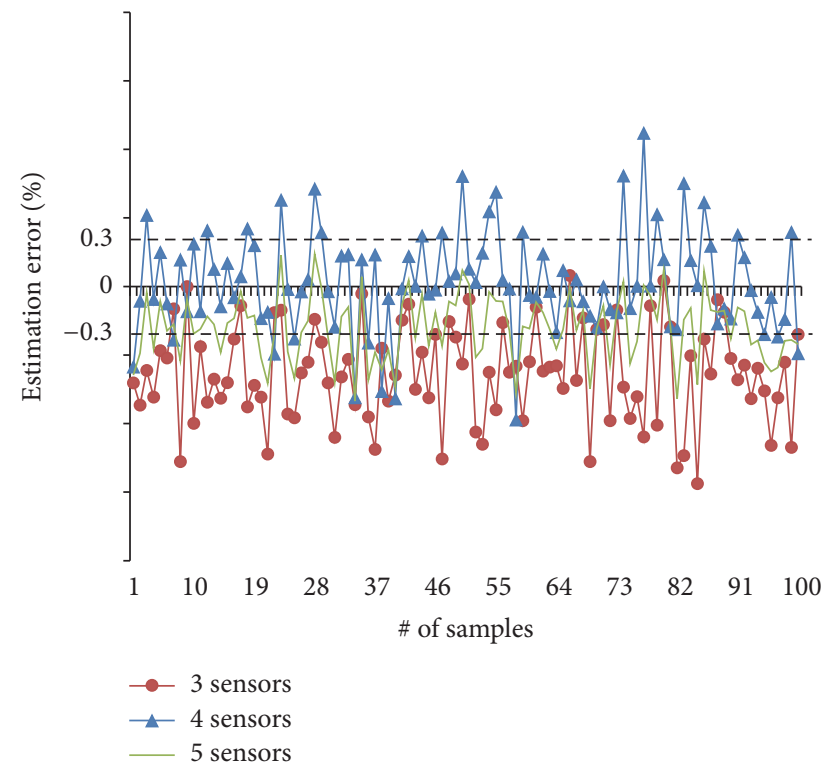

FIGURE 5: Estimation error with varying the number of sensors. object to determine if the object is human, instead of the position of the object. We propose a height estimation mechanism with low processing overhead. On the top of the mechanism, in this paper, we revise a compensation algorithm using interpolation method to reduce the estimation error of the height. Through implementation of our mechanism, we verify the performance of the height estimation. The experimental results indicate that our mechanism correctly and fast estimates the height of the object without complex computation. Through performance analysis, we show that the estimation accuracy is more affected by obstacle than the deployment of sensors or the number of sensors. For the future work, we plan to perform experiments with a practical scenario and to improve the estimation mechanism to compensate the estimation error or the environmental impact.

\section{Competing Interests}

The authors declare that they have no competing interests. 


\section{Acknowledgments}

This research was supported by the Sookmyung Women's University Research Grants (1-1603-2031) and by Basic Science Research Program through the National Research Foundation of Korea (NRF) funded by the Ministry of Education (NRF-2015R1D1A1A09057141).

\section{References}

[1] M. Walchli and T. Braun, "Building intrusion detection with a wireless sensor network," in Ad Hoc Networks, vol. 28 of Lecture Notes of the Institute for Computer Sciences, pp. 607-622, Springer, Berlin, Germany, 2010.

[2] R. Lara, D. Benítez, A. Caamaño, M. Zennaro, and J. L. RojoÁlvarez, "On real-time performance evaluation of volcanomonitoring systems with wireless sensor networks," IEEE Sensors Journal, vol. 15, no. 6, pp. 3514-3523, 2015.

[3] S. K. Dash, J. P. Sahoo, S. Mohapatra, and S. P. Pati, "Sensorcloud: assimilation of wireless sensor network and the cloud," in Advances in Computer Science and Information Technology Networks and Communications, vol. 84 of Springer Lecture Notes of the Institute for Computer Sciences, Social Informatics and Telecommunications Engineering, pp. 455-464, 2012.

[4] L. Buttyán, D. Gessner, A. Hessler, and P. Langendoerfer, "Application of wireless sensor networks in critical infrastructure protection: challenges and design options," IEEE Wireless Communications, vol. 17, no. 5, pp. 44-49, 2010.

[5] F. Viani, M. Salucci, P. Rocca, G. Oliveri, and A. Massa, "A multisensor WSN backbone for museum monitoring and surveillance," in Proceedings of the IEEE 6th European Conference on Antennas and Propagation (EUCAP '12), pp. 51-52, IEEE, Prague, Czech Republic, March 2012.

[6] T. Suzuki, A. Khan, M. Kobayashi, and W. Takita, "Collaboration in routing and velocity measurement function for mobile ad hoc networks," in Proceedings of the IEEE International Conference on Future Generation Communication and Networking (FGCN '07), pp. 108-113, Jeju Island, Korea, December 2007.

[7] E. Zamora and W. Yu, "Recent advances on simultaneous localization and mapping for mobile robots," IETE Technical Review, vol. 30, no. 6, pp. 490-496, 2013.

[8] J. Shim and Y. Lim, "Height estimation for applications in surveillance systems," IETE Technical Review, vol. 32, no. 5, pp. 321329, 2015.

[9] S. Samanta, P. U. Tembhare, and C. R. Pote, "A survey on 3D localization in wireless sensor networks," International Journal of Computational Engineering Research, vol. 3, no. 1, pp. 90-94, 2013.

[10] L. Wang, J. Zhang, and D. Cao, "A new 3-dimensional DV-Hop localization algorithm," Journal of Computational Information Systems, vol. 8, no. 6, pp. 2463-2475, 2012.

[11] H. Chen, P. Huang, M. Martins, H. C. So, and K. Sezaki, "Novel centroid localization algorithm for three-dimensional wireless sensor networks," in Proceedings of the International Conference on Wireless Communications, Networking and Mobile Computing (WiCOM '08), pp. 1-4, October 2008.

[12] E. Guerrero, H. Wang, J. Alvarez, and L. Rivero, "A threedimensional range-free localization algorithm based on mobile beacons for wireless sensor networks," Computer Aided Drafting, Design and Manufacturing, vol. 2010, no. 10, pp. 83-92, 2010.
[13] Q. Liu, P. Ren, and Z. Zhou, “Three-dimensional accurate positioning algorithm based on wireless sensor networks," Journal of Computers, vol. 6, no. 12, pp. 2582-2589, 2011.

[14] C. Liu, H. Jiang, and D.-L. Zeng, "Unitary matrix pencil algorithm for range-based 3D localization of wireless sensor network nodes," Journal of Networks, vol. 7, no. 9, pp. 1384-1390, 2012.

[15] Q. Shi, H. Huo, T. Fang, and D. Li, "A 3D node localization scheme for wireless sensor networks," IEICE Electronics Express, vol. 6, no. 3, pp. 167-172, 2009.

[16] Y. Wang and K. C. Ho, "An asymptotically efficient estimator in closed-form for 3-D AOA localization using a sensor network," IEEE Transactions on Wireless Communications, vol. 14, no. 12, pp. 6524-6535, 2015.

[17] H.-J. Shao, X.-P. Zhang, and Z. Wang, "Efficient closed-form algorithms for AOA based self-localization of sensor nodes using auxiliary variables," IEEE Transactions on Signal Processing, vol. 62, no. 10, pp. 2580-2594, 2014.

[18] D. M. Mitchell, "A heron-type formula for the reciprocal area of a triangle," Mathematical Gazette, vol. 89, no. 516, pp. 494-516, 2005.

[19] C. Suh, J.-E. Joung, and Y.-B. Ko, "New RF models of the TinyOS simulator for IEEE 802.15.4 standard," in Proceedings of the IEEE Wireless Communications and Networking Conference (WCNC '07), pp. 2238-2242, Hong Kong, March 2007.

[20] IEEE 802.15 WPAN ${ }^{\mathrm{TM}}$ Task Group, IEEE standard, http://www .ieee802.org/15/pub/TG4.html. 

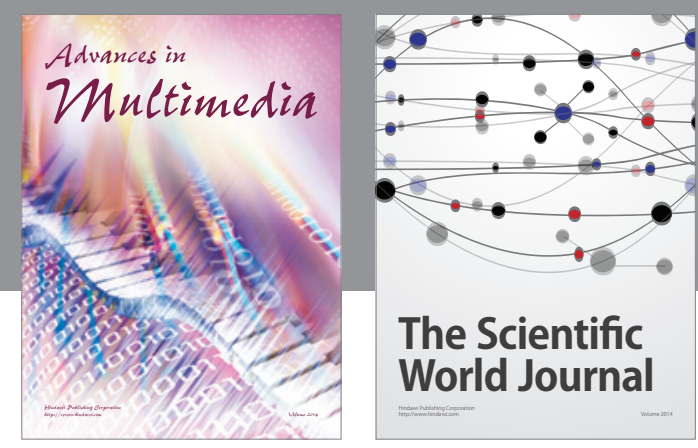

The Scientific World Journal
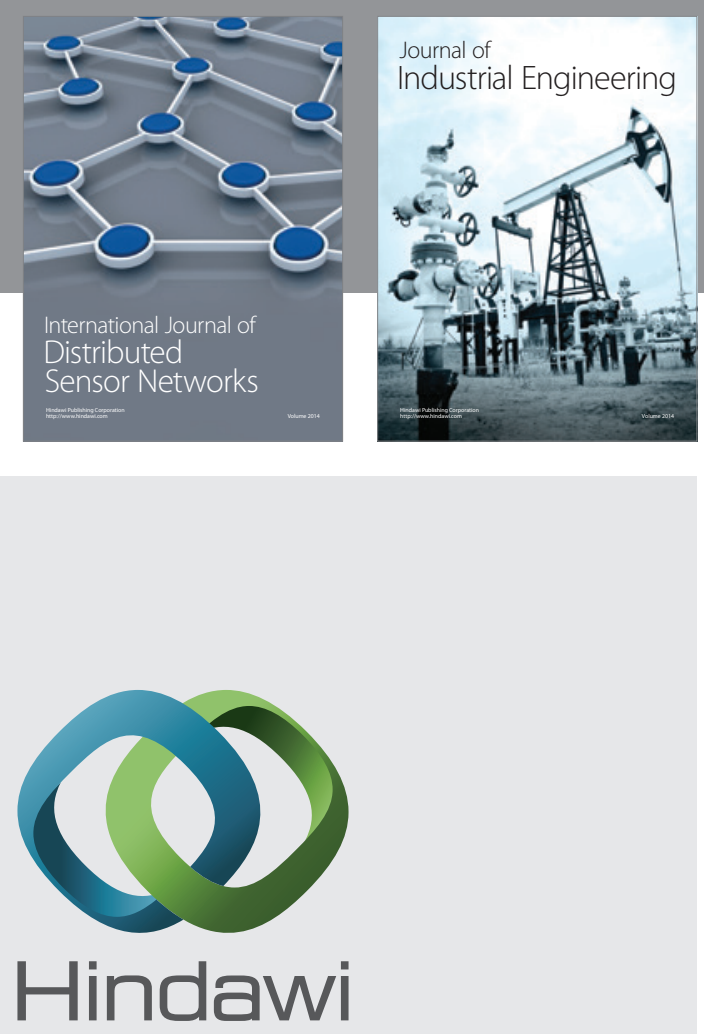

Submit your manuscripts at

http://www.hindawi.com

\section{Computer Networks} and Communications
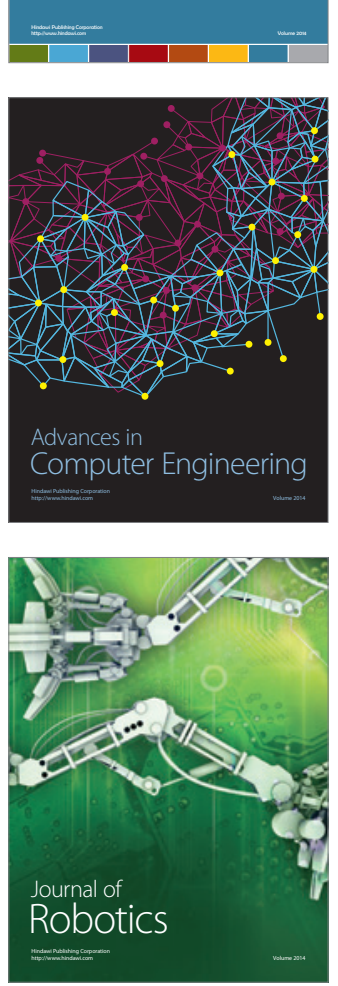
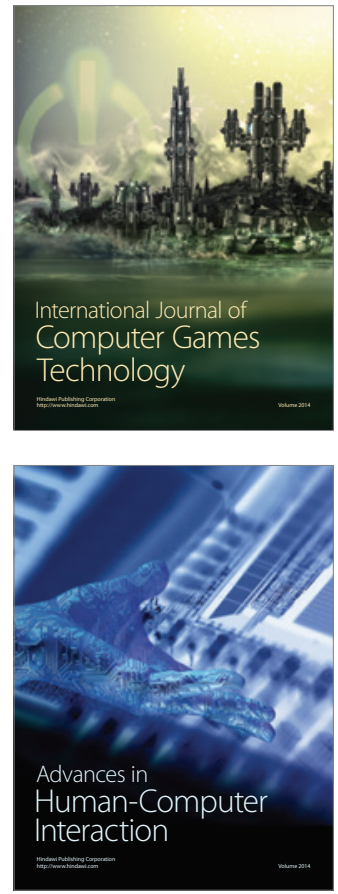
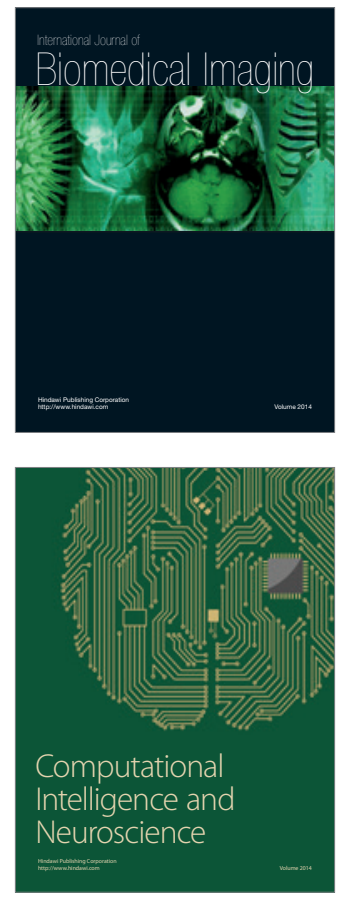
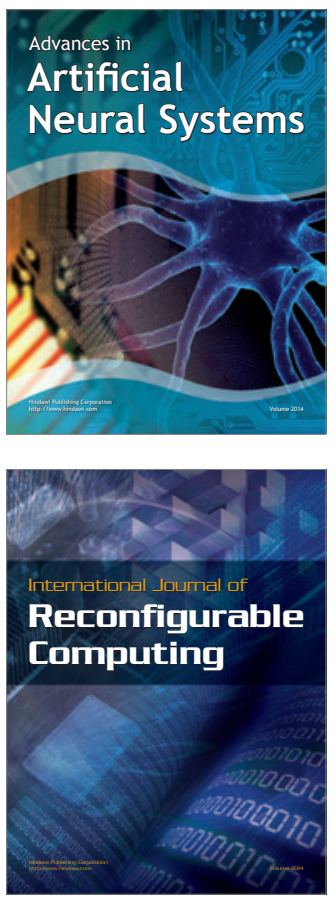
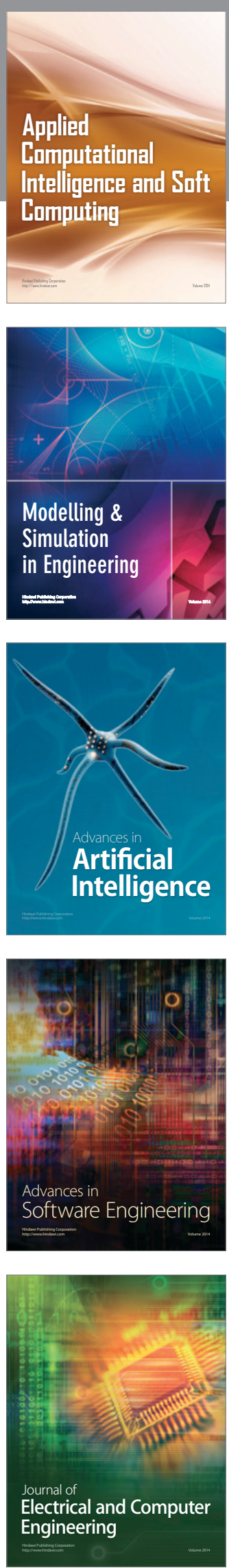